\title{
ARISE-a prospective, non-interventional, single-arm study assessing clinical parameters associated with the use of insulin degludec/insulin aspart in patients with type 2 diabetes in real-world settings: rationale and design
}

\author{
Gregory R. Fulcher (iD) ${ }^{1,2} \cdot$ Henrik Jarlov ${ }^{3} \cdot$ Johanne Spanggaard Piltoft $^{4} \cdot$ Kiran Pal Singh $^{5} \cdot$ Lei Liu $^{4} \cdot$ \\ Mafauzy Mohamed ${ }^{6} \cdot$ Nemencio Almare Nicodemus $\mathrm{Jr}^{7} \cdot$ Saleh Jaser Al-Jaser ${ }^{8} \cdot$ Adri Kok $^{9,10}$
}

Received: 12 June 2021 / Accepted: 16 September 2021 / Published online: 12 October 2021

(c) The Author(s), under exclusive licence to Springer Science+Business Media, LLC, part of Springer Nature 2021, corrected publication 2021

\begin{abstract}
Purpose IDegAsp, a co-formulation of long-acting basal (insulin degludec) and rapid-acting bolus (insulin aspart) insulin, provides separate prandial and basal glucose-lowering effects with relatively low risk of hypoglycaemia. Its efficacy and safety have been investigated in a large clinical trial programme (BOOST). We present the rationale and design of the ARISE study, which aims to assess glycaemic control and other clinical parameters associated with IDegAsp use in real world.

Methods ARISE is a 26-wk-long, prospective, non-interventional, single-arm study of patients with type 2 diabetes (T2D) initiating IDegAsp treatment. Approximately 1112 patients with T2D aged $\geq 18$ years previously on anti-hyperglycaemic drugs except IDegAsp will be enroled across six countries from 15 Aug 2019 to 12 Nov 2020. IDegAsp treatment will be initiated at the physicians' discretion and as per the local label. Key exclusion criteria include previous participation, or previous IDegAsp treatment. The primary and secondary endpoints are change in $\mathrm{HbA}_{1 \mathrm{c}}$ from baseline (wk 0 ) to study end (wk 26-36) and the proportion of patients achieving the target $\mathrm{HbA}_{1 \mathrm{c}}$ level of $<7 \%$ at the study end, respectively. A mixed model for repeated measurements will analyse the primary endpoint.

Conclusion Between-country differences in the prescription patterns of glucose-lowering agents in people with T2D warrant examination of their clinical use in different geographical settings. The ARISE study is designed to assess the clinical use of IDegAsp from real world in six different countries. Findings from the ARISE study will supplement those of previous randomised controlled studies by establishing real-world evidence of IDegAsp use in the participating countries.

Trial registration ClinicalTrials.gov, NCT04042441. Registered 02 August 2014, https://clinicaltrials.gov/ct2/show/ NCT04042441
\end{abstract}

Keywords Co-formulation $\cdot$ Insulin aspart $\cdot$ Insulin degludec $\cdot$ Real world $\cdot$ Type 2 diabetes

Supplementary information The online version contains supplementary material available at https://doi.org/10.1007/s12020021-02887-8.

Gregory R. Fulcher

greg.fulcher@sydney.edu.au

1 Department of Diabetes, Endocrinology and Metabolism, Royal North Shore Hospital, Sydney, NSW, Australia

2 Northern Clinical School, University of Sydney, Sydney, NSW, Australia

3 Novo Nordisk Pharma Gulf LLC, Dubai, UAE

4 Novo Nordisk A/S, Søborg, Denmark

5 Department of Endocrinology, Fortis Hospital, Mohali, Punjab, India
6 Department of Medicine, Hospital Universiti Sains Malaysia, Kota Bharu, Kelantan, Malaysia

7 Department of Biochemistry and Molecular Biology, University of the Philippines-College of Medicine, Manila, Philippines

8 Department of Internal Medicine, Specialised Medical Center, Riyadh, Saudi Arabia

9 Union and Clinton Hospitals in Alberton, Gauteng, South Africa

10 Department of Internal Medicine, University of the Witwatersrand, Johannesburg, South Africa 


\section{Introduction}

Type 2 diabetes (T2D) is a progressive disease characterised by insulin resistance and deterioration of beta-cell function over time. Sustained hyperglycaemia is associated with increased microvascular and macrovascular complications [1]. The American Diabetes Association (ADA) standard of care for patients with diabetes and the European Society for the Study of Diabetes recommend a reasonable glycated haemoglobin $\left(\mathrm{HbA}_{1 \mathrm{c}}\right)$ target of $<7.0 \%(53 \mathrm{mmol} / \mathrm{mol})$ and a stringent goal of $<6.5 \%$ ( $48 \mathrm{mmol} / \mathrm{mol}$ ) if achievable without the fear of hypoglycaemia or other adverse effects of treatment [2]. However, most patients with diabetes eventually fail to achieve the target glycaemic control with lifestyle modifications and oral anti-diabetes drugs (OADs) alone [3]. Treatment initiation and intensification with insulin is often the next step.

However, in practice, treatment intensification is often delayed or not performed due to treatment barriers (referred to as clinical inertia). These barriers include emphasis on the existing $\mathrm{HbA}_{1 \mathrm{c}}$ levels rather than on the long-term burden of hyperglycaemia, fear of hypoglycaemia, body weight gain, complex insulin regimens and financial Costs [3]. Different insulin regimens vary in their effects on glycaemic control, body weight, hypoglycaemia, and complexity. For example, in patients with T2D who failed to achieve target glycaemic control with OADs, the addition of basal or prandial insulin regimen led to overall similar reductions in $\mathrm{HbA}_{1 \mathrm{c}}$, but patients using basal insulin regimens achieved target glycaemic control with fewer hypoglycaemic episodes and less body weight gain than those using other insulin regimens [4]. However, basal insulin alone fails to provide prandial coverage and warrants intensification to a basal-bolus insulin regimen, which is complex [5]. Pre-mixed insulin regimens provide both basal and prandial coverage in one injection. Furthermore, they are simpler and more convenient than basal-bolus insulin regimens [6].

The current pre-mixed insulin formulations are designed to achieve both basal and mealtime glycaemic control. However, protamination of short-acting insulin results in intermediate-acting insulin, thereby failing to provide sustained basal coverage as observed with long-acting insulin. Furthermore, the protaminated fraction interferes with the soluble fraction, resulting in an undesirable shoulder effect of the short-acting insulin and potentially increasing the risk of hypoglycaemia at that time [6].

The co-formulation of insulin degludec (70\%) and insulin aspart (30\%) (IDegAsp) is available in a single pen. IDegAsp provides both long, flat and stable basal glucose-lowering effect and prandial insulin coverage. In addition, IDegAsp offers less complexity with fewer injections. IDegAsp does not require re-suspension, enabling reliable and accurate dosing [6]. The BOOST clinical investigation programme for patients with diabetes assessed the safety and efficacy of once or twice daily administration of IDegAsp using 26-wk-long treat-to-target studies. IDegAsp effectively improved glycaemic control and overcame most of the limitations associated with the use of pre-mix or basal-bolus insulin regimens and demonstrated a simpler titration regimen [7-11]. In a 38wk step-by-step intensification trial in patients with T2D, IDegAsp achieved similar efficacy and significantly fewer nocturnal hypoglycaemic episodes but with a significantly lower insulin dose and fewer injections than insulin glargine U100 and insulin aspart administered as a basal-bolus regimen (see Additional file 1) [12].

\section{Rationale}

Despite an extensive clinical development programme for IDegAsp, little evidence is available on the real-world use of this therapeutic option. Real-world studies are gaining importance due to their ability to assess the effectiveness and safety of new therapeutic agents in actual clinical settings. Despite being the gold standard for assessing the safety and efficacy of new therapeutic agents, randomised controlled trials (RCTs) lack generalisability of findings to a wider population. On the other hand, real-world studies generally include a wider population in the real-world setting due to broader patient inclusion criteria. As such, they complement the findings of RCTs [13]. The prevalence of diabetes and prescription patterns of glucose-lowering agents differs across countries. For example, the most preferred treatment routine is basal-bolus in Australia but pre-mixed insulin in Saudi Arabia $[14,15]$. Consequently, it is important to ascertain the clinical parameters associated with the use of IDegAsp in diverse geographical settings. In addition, it is important to understand the reasons for IDegAsp treatment initiation/discontinuation in various local settings. Therefore, the present prospective, real-world and non-interventional ARISE (A Ryzodeg Initiation and Switch Effectiveness) study was planned in six selected countries, i.e., Australia, India, Malaysia, Philippines, Saudi Arabia and South Africa.

Here, we present the rationale and design of the ARISE study that aims to assess glycaemic control and other clinical parameters associated with the use of IDegAsp in patients with T2D who were initiated with IDegAsp treatment in real-world settings across six countries, complementing the findings of RCTs on IDegAsp.

\section{Methods/Design}

\section{Study design}

ARISE is a prospective, multi-centre, open-label, noninterventional, single-arm study of IDegAsp in patients with 
Fig. 1 Study design

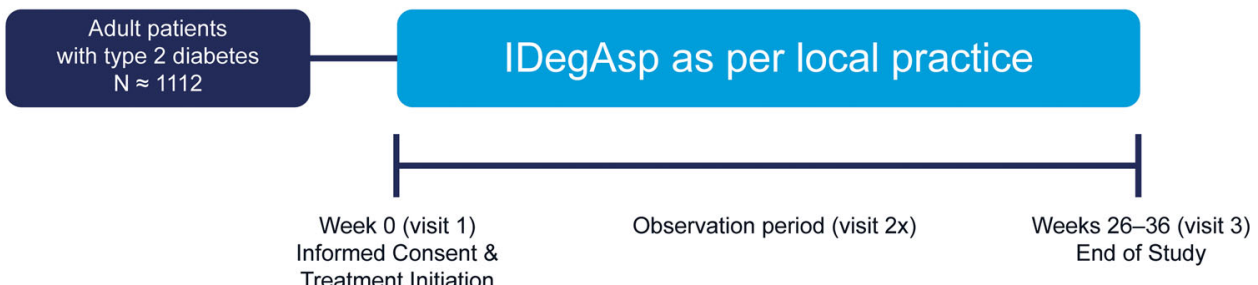

IDegAsp, co-formulation of insulin degludec (70\%) and insulin aspart (30\%)

T2D across Australia, India, Malaysia, Philippines, Saudi Arabia and South Africa. Adults with T2D using any glucose-lowering agents and who will be considered suitable for IDegAsp treatment at their physician's discretion will be enroled between 15 August 2019 and 12 November 2020. The duration of the study for each patient will be approximately 26 weeks (Fig. 1).

Patients will be included if a clinician, in conjunction with the patient/legal guardian, determines that IDegAsp is the most appropriate clinical choice and its use is consistent with the local label. Once the treatment course has been agreed upon, the patient could be approached to participate in the study. The initial dose or any further dose adjustments are determined by the treating physician. During the study, insulin dose adjustments or addition/discontinuation of any glucose-lowering agents including IDegAsp is at the treating physician's discretion based on usual clinical decision-making.

\section{Study population}

Male and female patients aged $\geq 18$ years initiated on IDegAsp treatment will be eligible to be enroled in the study if they were diagnosed with T2D and treated with any anti-hyperglycaemic agent (apart from IDegAsp) in the past 26 weeks and had $\mathrm{HbA}_{1 \mathrm{c}}$ level measured $\leq 12$ wk prior to participation. Exclusion criteria include prior participation in the study, previous IDegAsp treatment, mental incapacity, unwillingness to participate or language barriers that would lead to inadequate understanding or cooperation.

\section{Data collection}

Following eligibility assessment and receipt of informed consent, patient-level data will be collected prospectively for approximately 26 weeks during patients' routine clinical visits. Data will be collected at the treatment initiation visit (v1, wk 0), at multiple intermediate visits depending on the local clinical practice ( $\mathrm{v} 2 \times$, wk $1-25)$, at the end of the study visit (v3, any first visit within wk 26-36) and at treatment discontinuation visit ( $\mathrm{v} 3 \times$, during wk 0-36), if applicable.

\section{Study assessments}

Details on patients' relevant medical history including a history of cardiovascular disease and presence of cardiovascular risk factors (e.g., hypertension, dyslipidaemia and renal disease) will be collected retrospectively from medical records. T2D diagnosis date, treatment and complications will be recorded. Details of antidiabetic medication (including treatments stopped prior to IDegAsp initiation, e.g., insulins, GLP-1 RAs and/or OADs) must be recorded at visit 1 . Any changes to the antidiabetic medication must be recorded at all subsequent visits and the eCRF should be updated accordingly. The information collected includes tradename or generic name, start date (only start year is applicable if more than one year), dose, frequency of administration, primary indication and stop date or continuation (yes/no). Data will also be collected about the presence of hypoglycaemic risk factors defined as follows: patient experiencing $\geq 1$ severe hypoglycaemic episodes within the past year, moderate or severe estimated glomerular filtration rate of $<59 \mathrm{~mL} / 1.73 \mathrm{~m}^{2}$, hypoglycaemic symptom unawareness (history of impaired autonomic responses) and exposure to insulin treatment for $\geq 5$ years. The reasons for initiation and potential discontinuation of IDegAsp treatment will be noted. Patient-reported hypoglycaemic episodes including non-severe [episode with symptoms and/or self-measured blood glucose level of $\leq 3.9 \mathrm{mmol} / \mathrm{L}(<70 \mathrm{mg} / \mathrm{dL})$ within the past $4 \mathrm{wk}$ ], nocturnal (occurred between midnight and early morning) and severe (episode requiring assistance of a third person for a corrective action within the past 4 and 26 weeks) episodes as defined by a work group of the ADA and the Endocrine Society will be recorded [16]. Patients will be asked to recall all episodes of non-severe hypoglycaemia in the last 4 weeks and all episodes of severe hypoglycaemia within the last 4 and 26 weeks. The number of non-severe episodes and severe episodes will be recorded in the eCRF. Glucose levels will be measured by the laboratory. The treating physicians or appropriately qualified and trained delegates will enter data from patients' medical records into the eCRF. Diabetes-related healthcare resource utilisation associated with severe hypoglycaemia and with management of diabetes and diabetes-related complications will be 
Table 1 Assessments at each visit during the study, if available

\begin{tabular}{|c|c|c|c|c|}
\hline $\begin{array}{l}\text { Study week } \\
\text { Study day (visit window) } \\
\text { Visit description }\end{array}$ & $\begin{array}{l}0 \\
\text { Visit } 1 \\
\text { Informed consent and } \\
\text { treatment initiation }\end{array}$ & $\begin{array}{l}1-25 \\
\text { Visit } 2 \times \\
\text { Intermediate visits }\end{array}$ & $\begin{array}{l}26-36 \\
\text { Visit } 3 \\
\text { End of study }\end{array}$ & $\begin{array}{l}0-36 \\
\text { Visit } 3 \times \\
\text { Treatment discontinuation }\end{array}$ \\
\hline \multicolumn{5}{|l|}{ Patient and treatment-related assessments ${ }^{\mathrm{b}}$} \\
\hline Informed consent ${ }^{\mathrm{c}}$ & $\checkmark$ & & & \\
\hline Inclusion/exclusion criteria & $\checkmark$ & & & \\
\hline Demographics & $\checkmark$ & & & \\
\hline Body weight & $\sigma^{\mathrm{d}}$ & $\checkmark$ & $\mathfrak{J}^{\mathrm{e}}$ & $\checkmark$ \\
\hline Height & $d^{\mathrm{d}}$ & & & \\
\hline Systolic and diastolic BP & $\checkmark$ & & $\mathfrak{J}^{\mathrm{e}}$ & $\checkmark$ \\
\hline Medical history ${ }^{\mathrm{f}}$ & $\checkmark$ & & & \\
\hline Diabetes history (diagnosis, duration and complications) ${ }^{g}$ & $\checkmark$ & & & \\
\hline Presence of hypoglycaemic risk factors ${ }^{\mathrm{h}}$ & $\checkmark$ & & & \\
\hline Concomitant medication ${ }^{\mathrm{i}}$ & $\checkmark$ & & & \\
\hline Insulin/OAD/GLP-1 RA details prior to IDegAsp initiation & $\checkmark$ & & & \\
\hline Date of IDegAsp initiation & $\checkmark$ & & & \\
\hline Reason for IDegAsp initiation & $\checkmark$ & & & \\
\hline Dose and frequency of IDegAsp & $\checkmark$ & $\checkmark$ & $\checkmark$ & $\checkmark$ \\
\hline $\begin{array}{l}\text { Number of weekly SMPG measurement (in the past } 4 \mathrm{wk} \\
\text { overall) }\end{array}$ & $\checkmark$ & & $\checkmark$ & $\rho^{\mathrm{j}}$ \\
\hline $\begin{array}{l}\text { Reasons and date of discontinuation of IDegAsp, if } \\
\text { applicable }\end{array}$ & & & & $\checkmark$ \\
\hline \multicolumn{5}{|c|}{ Treatment effect, hypoglycaemia, safety assessments ${ }^{\mathrm{b}}$ and end of study } \\
\hline $\mathrm{HbA}_{1 \mathrm{c}}$ (local laboratory measured) & $\Omega^{\mathrm{d}}$ & $\checkmark$ & $\boldsymbol{J}^{\mathrm{e}}$ & $\checkmark$ \\
\hline FPG (local laboratory measured) & $d^{\mathrm{d}}$ & $\checkmark$ & $\boldsymbol{J}^{\mathrm{e}}$ & $\checkmark$ \\
\hline $\begin{array}{l}\text { Patient-reported non-severe hypoglycaemic episodes in the } \\
\text { past } 4 \mathrm{wk} \text { (overall and nocturnal) }\end{array}$ & $\checkmark$ & & $\checkmark$ & $\jmath^{\mathrm{j}}$ \\
\hline $\begin{array}{l}\text { Patient-reported severe hypoglycaemic episodes in the past } \\
26 \text { wk (overall) }\end{array}$ & $J^{\mathrm{k}}$ & & $J^{\mathrm{k}}$ & $\boldsymbol{J}^{\mathrm{j}, \mathrm{k}}$ \\
\hline $\begin{array}{l}\text { Resource utilisation associated with diabetes management } \\
\text { (in the past } 12 \mathrm{wk} \text { ) }\end{array}$ & $\checkmark$ & & $\checkmark$ & $\boldsymbol{J}^{\mathrm{k}}$ \\
\hline $\begin{array}{l}\text { Resource utilisation associated with severe hypoglycaemia } \\
\text { (in the past } 26 \mathrm{wk} \text { ) }\end{array}$ & $\checkmark$ & & $\checkmark$ & $J^{\mathrm{b}}$ \\
\hline Pregnancy & $\checkmark$ & $\checkmark$ & $\checkmark$ & $\checkmark$ \\
\hline Adverse episodes & & $\checkmark$ & $\checkmark$ & $\checkmark$ \\
\hline End of study & & & $\checkmark$ & \\
\hline
\end{tabular}

$B P$ blood pressure, $F P G$ fasting plasma glucose, GLP-1 RA glucagon-like peptide-1 receptor agonist, HbAlc glycated haemoglobin, IDegAsp insulin degludec/insulin aspart, $N S$ non-significant, $O A D$ oral antidiabetic drug, $S M P G$ self-measured plasma glucose

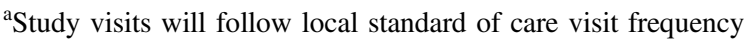

${ }^{\mathrm{b}}$ Assessments will be collected as available

${ }^{c}$ Informed consent will be obtained before any study-related activities

${ }^{\mathrm{d}}$ Baseline assessments must not be performed $>12$ wk prior to signing informed consent

${ }^{\mathrm{e}}$ First value recorded during the end-of-study visit window is accepted

${ }^{\mathrm{f}} \mathrm{A}$ medical event that a patient experienced in the past

${ }^{\mathrm{g}}$ Diabetes history (date of diagnosis) and diabetes-related complications (retinopathy, neuropathy and nephropathy) will be recorded

${ }^{\mathrm{h}}$ Hypoglycaemic risk factors will be collected as follows: if the patient experienced at least one severe hypoglycaemic episode in the past 1 year, moderate or severe chronic renal failure or estimated glomerular filtration rate of $<59 \mathrm{~mL} / \mathrm{min} / 1.73 \mathrm{~m}^{2}$, if the patient is unaware of hypoglycaemic symptoms as judged by the physician or if the patient is exposed to insulin treatment for $\geq 5$ years

${ }^{\mathrm{i}}$ Anti-diabetic drugs or any glucose-lowering agents other than IDegAsp

${ }^{\mathrm{j}}$ Data will be collected based on patient recall. In the case of discontinuation, data will be based on the period since the time of visit

${ }^{\mathrm{k}}$ The number of patient-reported severe hypoglycaemic episodes in the past $4 \mathrm{wk}$ (overall) will also be collected

recorded. No additional diagnostic or monitoring procedures beyond local standard clinical practice will be performed for the study patients. The assessments to be performed at each visit are described in Table 1.
Data will be obtained via electronic primary data collection and continue until the end of the study. Data will be collected for all patients including those who discontinue IDegAsp, unless the patient withdraws informed consent. 
Patients can withdraw at any stage of the study. If a patient discontinues treatment, the primary reason will be recorded.

\section{Study endpoints}

The primary endpoint is change in $\mathrm{HbA}_{1 \mathrm{c}}$ from baseline (week 0) to the end of study (the first visit within the window of wk 26-36). The secondary endpoints include change in fasting plasma glucose level and body weight from baseline to the end of the study. A full list of the study endpoints is presented in Table 2.

\section{Data management}

Physicians will ensure that study-specific data are captured in an eCRF and that data are transferred electronically to a secure virtual data centre to protect patient identity. The system for electronic data capture will be provided and maintained by Oracle under the supervision of Novo Nordisk.

\section{Administrative structure of the study}

The study is sponsored by Novo Nordisk. A contract research organisation will monitor the study procedures and conduct periodic monitoring either via telephone or site visits for compliance with the protocol and regulatory guidelines (Good Pharmacoepidemiology Practice, Good Pharmacovigilance Practice Module VI and Ethical Guidelines for Medical and Health Research Involving Human Subjects). The treating physician and institution will allow direct access to source documents for verification by Novo Nordisk monitors or its agents and appropriate regulatory authorities.

The study will be conducted in agreement with the approved protocol and the ethical principles laid down in the Declaration of Helsinki, in accordance with the International Council on Harmonisation of Technical Requirements for Registration of Pharmaceuticals for Human Use. Prior to study initiation, critical documents such as regulatory approval/ notification, physician's medical qualification, approved final protocol and approvals from various institutional review committees will be made available to the sponsor.

\section{Statistical methods and sample size}

\section{Sample size}

The sample size calculation is based on the primary endpoint analysis and aims to have sufficient power for primary endpoint analysis, both overall and in each of the six participating countries. The phase 3 a programme of IDegAsp included patients with different medication background that
Table 2 Study endpoints

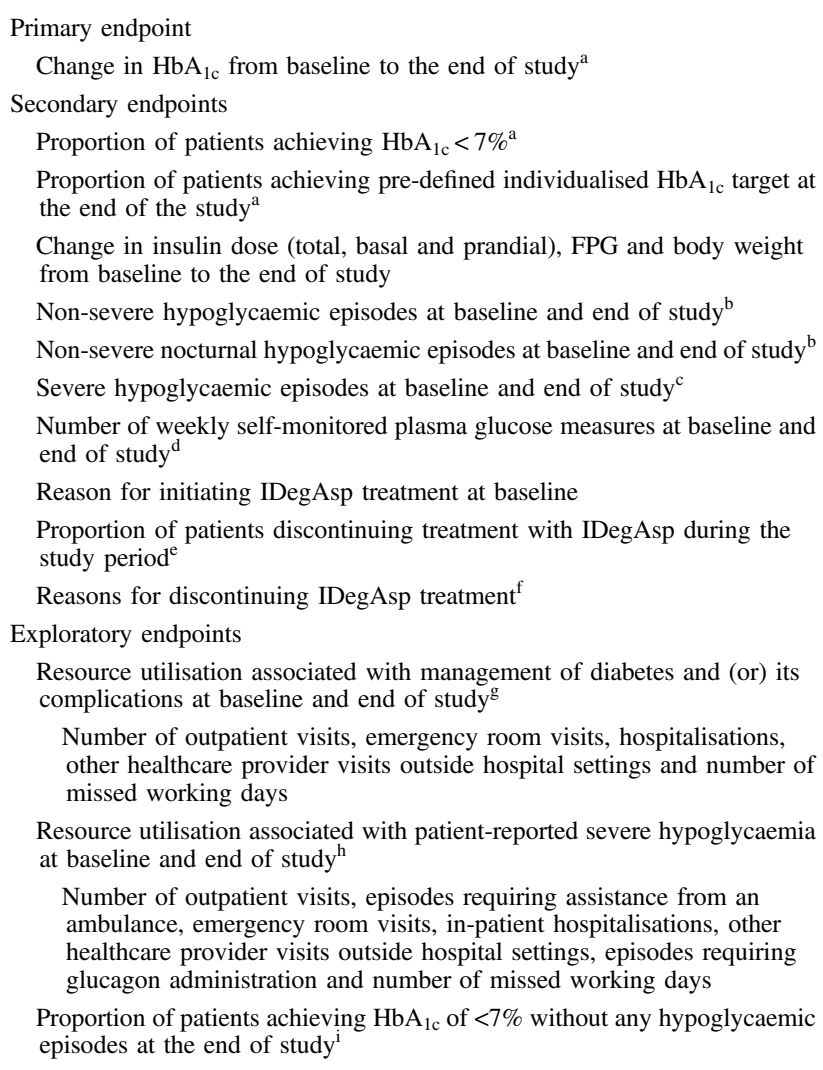

Outpatient visit is defined as medical treatment of a patient who is not hospitalised overnight but who visits a hospital, clinic or associated facility for diagnosis or treatment

In-patient hospitalisation is defined as medical treatment that is provided in a hospital or other facility and requires atleast one overnight stay

Emergency room visit is defined as a visit to a hospital area equipped and staffed for the prompt treatment of acuteillness, trauma or other medical emergencies. dOnly applies to resource utilisation associated with patient-reportedsevere hypoglycaemia

FPG fasting plasma glucose, HbAlc glycated haemoglobin, IDegAsp insulin degludec/insulin aspart

${ }^{a}$ End of study is any first visit within wk 26-36

${ }^{\mathrm{b}}$ Episodes occurring within $4 \mathrm{wk}$ prior to initiation of IDegAsp treatment and within 4 wk prior to the end of study

${ }^{c}$ Episodes occurring within 26 wk prior to IDegAsp treatment initiation and within $26 \mathrm{wk}$ prior to the end of study

${ }^{\mathrm{d}}$ Measurements during 7 days prior to initiation of IDegAsp treatment and 7 days prior to the end of study

${ }^{\mathrm{e}}$ At treatment discontinuation (wk 0-26) or at the end of study

${ }^{\mathrm{f}}$ At treatment discontinuation (wk 0-36)

${ }^{\mathrm{g}}$ Visits and contracts within $12 \mathrm{wk}$ prior to initiation of IDegAsp treatment and within 4 wk prior to the end of study (wk 26-36)

${ }^{\mathrm{h}}$ Episodes occurring within $26 \mathrm{wk}$ prior to initiation of IDegAsp treatment and $26 \mathrm{wk}$ prior to the end of study

${ }^{i}$ Episodes occurring within 4 wk prior to the end of study. FPG, fasting plasma glucose; $\mathrm{HbA}_{1 \mathrm{c}}$, glycated haemoglobin; IDegAsp, insulin degludec/insulin aspart. 
ranged from OADs to basal only or pre-mix insulins. Most previous phase $3 \mathrm{a}$ studies were treat-to-target in nature; therefore, dose up-titration was allowed and reductions in $\mathrm{HbA}_{1 \mathrm{c}}$ levels ranged from 1.2 to $3.3 \%$. However, the noninterventional set-up of the present study does not allow controlled titration and that criteria for specific prior antidiabetes treatment are not included. Hence, we do not expect similar changes in $\mathrm{HbA}_{1 \mathrm{c}}$ after IDegAsp treatment as seen in previous phase 3 a trials. Therefore, a conservative approach of an expected mean (SD) difference of $0.5 \%$ $(1.8 \%)$ in $\mathrm{HbA}_{1 \mathrm{c}}$ is applied.

To be able to detect this mean difference with $90 \%$ power, each country should have a sample size of 139 patients available for analysis. Assuming approximately $25 \%$ of patients will have missing $\mathrm{HbA}_{1 \mathrm{c}}$ measurement at the end of the study, a total (from all countries) of 1112 patients will be enroled to ensure a sufficient sample for the primary endpoint analysis overall and in each participating country.

\section{Statistical analysis}

All analyses will be performed overall as well as by each country and by previous anti-hyperglycaemic treatment [OADs only, basal only ( \pm OADs), glucagon-like peptide- $1 \pm$ insulin ( \pm OADs), basal-bolus $( \pm$ OADs) and pre-mix/selfmixed insulin \pm bolus ( \pm OADs)]. Statistical analyses for the primary and secondary endpoints will be performed as twosided tests with a significance level of 0.05 . No adjustments will be made for multiple comparisons. Descriptive statistics (mean, SD, median and range for continuous variables and proportion for categorical variables) will be used to describe baseline characteristics of patients. The full analysis set (FAS) which includes all eligible patients who provided informed consent and initiated IDegAsp treatment will be used to analyse all endpoints. The primary analysis of the primary endpoint resembles an intention-to-treat-like approach and will be performed based on the FAS with a mixed model for repeated measurements. The secondary analysis of the primary endpoint is also based on FAS and will be similar to the primary analysis, except that patients discontinuing IDegAsp will be censored at the date of discontinuation but contribute with data until treatment discontinuation. In addition, the robustness of the estimate from the primary analysis will be assessed with additional adjustment for other confounders a priori believed to have an influence on change in $\mathrm{HbA}_{1 \mathrm{c}}$; e.g., concomitant anti-hyperglycaemic medication(s) and Ramadan (in relevant countries). Study sites will also be included in the model to account for within-site correlation.

\section{Patient and public involvement}

Patients and/or the public were not involved in the design, or conduct, or reporting or dissemination plans of this research.

\section{Discussion}

The global prevalence of diabetes is increasing and is expected to affect 578 million individuals by 2030. Highincome and developed countries have largely contributed to the prevalence data due to better healthcare systems, accurate diagnosis and treatment affordability. Nevertheless, by 2045, the largest increase in prevalence is expected from regions that are moving out from low- to middle-income status. The national prevalence of diabetes varies across the six participating countries and ranges from $6.3 \%$ in Philippines to $18.3 \%$ in Saudi Arabia [17]. Various studies that assessed insulin treatment initiation and intensification patterns in patients with T2D confirmed the presence of clinical inertia in the participating countries. In most of these six participating countries, it took approximately 10 years of disease duration and $\mathrm{HbA}_{1 \mathrm{c}}$ levels of $9.0-10.0 \%$ before insulin treatment was initiated. Furthermore, most insulin regimens remained unchanged/not intensified despite high $\mathrm{HbA}_{1 \mathrm{c}}$ levels [18-21].

IDegAsp is the first co-formulation of long-acting basal insulin with a rapid-acting mealtime insulin in one pen approved for use in patients with type 1 diabetes and T2D. It addresses some of the clinical and non-clinical challenges associated with initiating and intensifying insulin treatment such as complex/inflexible regimen, fear of hypoglycaemia, weight gain, higher insulin dose and poor patient treatment adherence [6]. However, there is paucity of real-world data regarding the clinical use of IDegAsp. The ARISE study is designed to investigate glycaemic control and other clinical parameters associated with the use of IDegAsp in real-world settings and the study will enable data collection reflecting routine clinical practice in the six participating countries. Furthermore, inclusion of the proportion of patients achieving $\mathrm{HbA}_{1 \mathrm{c}}<7 \%$ without hypoglycaemia as an exploratory endpoint would help clinicians in selecting better treatment care in this population.

However, the study also has several limitations that deserve mention. Unlike in RCTs, the data collected in this study will reflect routine clinical practice rather than mandatory assessments at prespecified time points, which may impact the amount of data collected and its interpretation. The broad study inclusion criteria will enable inclusion of a T2D population reflecting real-world clinical practice. However, the study population may still be subject to inclusion bias. For example, patients who are either highly motivated or, conversely, difficult to treat with the currently available treatment modalities are more likely to be enroled. Furthermore, a high level of heterogeneity among the participating countries in terms of clinical practice and insurance coverage is expected, which could potentially compromise the external validity of the overall study results across participating countries. This will be mitigated partially by country-level analyses and 
wherein the representativeness of the study population within each country may be assessed with respect to demographics and clinical information. The primary analysis includes all patients who are initiating with IDegAsp treatment irrespective of potential treatment discontinuation during the study and should hence be representative of the target population. However, potential attrition bias may occur if there are differences in glycaemic changes between patients who withdraw from the study and those who continue to be in the study. A mixed model for repeated measurement, which takes into account data points until time of withdrawal, will minimise the influence of such attrition bias; however, data of patients who continue to be in the study will still drive the overall results.

Another key limitation of this study is the single-arm design. With the absence of a comparator arm, alternative explanations for changes from baseline in evaluated endpoints cannot be ruled out. Placebo effects are unaccounted for and may contribute to changes in observed clinical parameters following IDegAsp treatment. Likewise, regression towards the mean may lead to overestimation of the observed changes. Further, an observed glycaemic change may be biased by the clinical reason to initiate IDegAsp treatment and other sources of bias that are unaccounted for. Above considerations are all understood as inherent limitations in the context of a single-arm study design. Finally, an impact of the COVID-19 pandemic outbreak during the study period cannot be ruled out. To minimise any impact of COVID19 , additional 'end of the study visits' after the actual end of the study visit window were included and two sensitivity analyses were planned for the primary endpoint (data within the end of the study visit window and data from all visits).

The results of the ARISE study will nevertheless provide insights into clinical parameters associated with the use of IDegAsp in clinical practice across six different countries and hence a supplement and real-world contextualisation to the existing evidence base on IDegAsp.

\section{Data availability}

The protocol is available from the corresponding author on reasonable request.

Acknowledgements The authors would like to acknowledge Dr. Shahid Akhtar for his critical review of this manuscript and contribution to the background research. The authors would also like to acknowledge Dr. Anil Dandu, M. Pharm, PhD, from Novo Nordisk Global Business Services, Bengaluru, India, for his medical writing assistance in developing this manuscript and Enago Life Sciences for their language editing support.

Author contributions G.R.F., H.J., J.S.P. and L.L. contributed to conception, study design and development of the study protocol. K.P.S. contributed to conception and study design. G.R.F., K.P.S. and
M.M. were involved in the recruitment of patients. J.S.P. contributed to development of the statistical analytical plan. G.R.F. is the study chief investigator and M.M. is the lead investigator for Malaysia. All authors critically reviewed and participated in manuscript revision and approved the final draft.

Funding This study is funded by Novo Nordisk (Grant Number - Not available).

\section{Compliance with ethical standards}

Conflict of interest G.R.F. has received honoraria, teaching and research sponsorship/grants from AstraZeneca, Boehringer Ingelheim, Janssen, MSD, Novartis, Novo Nordisk and Sanofi-Aventis. H.J., J.S.P. and L.L. are employees of and hold shares at Novo Nordisk. K.P.S. has no conflicts of interests to disclose. M.M. received research contracts from Novo Nordisk and Eli Lilly and is a member of the local advisory board for Novo Nordisk, Eli Lilly and Sanofi. N.A.N. is a member of the local advisory board of Novo Nordisk, AstraZeneca, Merck and Torrent and received speaker honoraria from Novo Nordisk, AstraZeneca, Merck, Torrent, Eli Lilly, Sanofi, LRI-Therapharma and Servier. S.J.A.-J. has no conflicts of interests to disclose. A.K. has been involved in various advisory boards for Abbott, Adcock Ingram, AstraZeneca, Bristol-Myers Squibb, Boehringer Ingelheim, Eli Lilly, Janssen Pharmaceuticals, Merck, Merck Sharp \& Dohme, Mundipharma, Novartis, Novo Nordisk, Pfizer and Sanofi and is a speaker panel member for Abbott, Aspen-GlaxoSmithKline, AstraZeneca, Bristol-Myers Squibb, Boehringer Ingelheim, Eli Lilly, Janssen Pharmaceuticals, Merck, Merck Sharp \& Dohme, Novartis, Novo Nordisk, Pharmaplan, Pfizer and Sanofi and is involved in research with AstraZeneca, Merck Sharp \& Dohme, Novo Nordisk and Pfizer. A.K. has received speaker or consulting honoraria from Novo Nordisk, Eli Lilly, AstraZeneca, Boehringer Ingelheim, Merck Sharp \& Dohme, Sanofi, Amgen, Abbott, GlaxoSmithKline and Servier. A.K. served as a consultant and speaker panel member for Novo Nordisk.

Consent to participate Written informed patient consent will be obtained prior to participation in the study.

Ethics approval The study results will be communicated through abstract submission and presentation at national and international conferences and will be submitted for publication in peer-reviewed scientific and medical journals. Approvals from Institutional Review Board (IRB)/Independent Ethics Committee (IEC) (or other appropriate bodies as required locally) were obtained prior to study initiation.

Publisher's note Springer Nature remains neutral with regard to jurisdictional claims in published maps and institutional affiliations.

\section{References}

1. V.A. Fonseca, Defining and characterizing the progression of type 2 diabetes. Diabetes Care 32, S151-S156 (2009)

2. S.E. Inzucchi, R.M. Bergenstal, J.B. Buse, M. Diamant, E. Ferrannini, M. Nauck, A.L. Peters, A. Tsapas, R. Wender, D.R. Matthews, Management of hyperglycemia in type 2 diabetes, 2015: a patient-centered approach: update to a position statement of the American Diabetes Association and the European Association for the Study of Diabetes. Diabetes Care 38, 140-149 (2015) 
3. K. Khunti, D. Millar-Jones, Clinical inertia to insulin initiation and intensification in the UK: a focused literature review. Prim Care Diabetes 11, 3-12 (2017)

4. R.R. Holman, A.J. Farmer, M.J. Davies, J.C. Levy, J.L. Darbyshire, J.F. Keenan, S.K. Paul, Three-year efficacy of complex insulin regimens in type 2 diabetes. N. Engl. J. Med. 361, 1736-1747 (2009)

5. G. Grunberger, D. Sze, A. Ermakova, R. Sieradzan, T. Oliveria, E. M. Miller, Treatment intensification with insulin pumps and other technologies in patients with type 2 diabetes: results of a physician survey in the United States. Clin. Diabetes 38, 47-55 (2020)

6. S. Atkin, Z. Javed, G. Fulcher, Insulin degludec and insulin aspart: novel insulins for the management of diabetes mellitus. Ther. Adv. Chronic Dis. 6, 375-388 (2015)

7. G.R. Fulcher, J.S. Christiansen, G. Bantwal, M. Polaszewska-Muszynska, H. Mersebach, T.H. Andersen, L.K. Niskanen, Comparison of insulin degludec/insulin aspart and biphasic insulin aspart 30 in uncontrolled, insulin-treated type 2 diabetes: a phase $3 \mathrm{a}$, randomized, treat-to-target trial. Diabetes Care 37, 2084-2090 (2014)

8. S. Kaneko, F. Chow, D.S. Choi, S. Taneda, K. Hirao, Y. Park, T. H. Andersen, M.A. Gall, J.S. Christiansen, Insulin degludec/ insulin aspart versus biphasic insulin aspart 30 in Asian patients with type 2 diabetes inadequately controlled on basal or pre-/selfmixed insulin: a 26-week, randomised, treat-to-target trial. Diabetes Res. Clin. Pract. 107, 139-147 (2015)

9. S. Kumar, H.C. Jang, N.G. Demirag, T.V. Skjøth, L. Endahl, B. Bode, Efficacy and safety of once-daily insulin degludec/insulin aspart compared with once-daily insulin glargine in participants with Type 2 diabetes: a randomized, treat-to-target study. Diabet. Med. 34, 180-188 (2017)

10. Y. Onishi, Y. Ono, R. Rabol, L. Endahl, S. Nakamura, Superior glycaemic control with once-daily insulin degludec/insulin aspart versus insulin glargine in Japanese adults with type 2 diabetes inadequately controlled with oral drugs: a randomized, controlled phase 3 trial. Diabetes Obes. Metab. 15, 826-832 (2013)

11. W. Yang, J. Ma, T. Hong, M. Liu, H. Miao, Y. Peng, C. Wang, X. Xu, T. Yang, A.M. Nielsen, L. Pan, Efficacy and safety of insulin degludec/insulin aspart versus biphasic insulin aspart 30 in Chinese adults with type 2 diabetes: A phase III, open-label, 2:1 randomized, treat-to-target trial. Diabetes Obes. Metab. 21, $1652-1660$ (2019)
12. A. Philis-Tsimikas, K. Astamirova, Y. Gupta, A. Haggag, D. Roula, B.A. Bak, E.G. Fita, A.M. Nielsen, T. Demir, Similar glycaemic control with less nocturnal hypoglycaemia in a 38week trial comparing the IDegAsp co-formulation with insulin glargine U100 and insulin aspart in basal insulin-treated subjects with type 2 diabetes mellitus. Diabetes Res. Clin. Pract. 147, 157-165 (2019)

13. L. Blonde, K. Khunti, S.B. Harris, C. Meizinger, N.S. Skolnik, Interpretation and impact of real-world clinical data for the practicing clinician. Adv. Ther. 35, 1763-1774 (2018)

14. G. Deed, G. Kilov, T. Dunning, R. Cutfield, J. Overland, T. Wu, Use of 50/50 premixed insulin analogs in type 2 diabetes: systematic review and clinical recommendations. Diabetes Ther. 8, 1265-96 (2017)

15. A. Chaudhary, Insulin prescribing practices in Saudi Arabia. World. World J. Pharm. Pharm. Sci. 4, 29-40 (2015)

16. E.R. Seaquist, J. Anderson, B. Childs, P. Cryer, S. Dagogo-Jack, L. Fish, S.R. Heller, H. Rodriguez, J. Rosenzweig, R. Vigersky, Hypoglycemia and diabetes: a report of a workgroup of the American Diabetes Association and the Endocrine Society. J. Clin. Endocrinol. Metab. 98, 1845-1859 (2013)

17. International Diabetes Federation, IDF Diabetes Atlas.: https://www.diabetesatlas.org/upload/resources/2019/IDF_Atlas_ 9th_Edition_2019.pdf (2019). Accessed 17 Mar 2020

18. T.M. Davis, W.A. Davis, D.G. Bruce, Glycaemic levels triggering intensification of therapy in type 2 diabetes in the community: the Fremantle Diabetes Study. Med. J. Aust. 184, 325-328 (2006)

19. A. Jabbar, K. Abdallah, A. Hassoun, R. Malek, C. Senyucel, E. Spaepen, T. Treuer, I. Bhattacharya, Patterns and trends in insulin initiation and intensification among patients with Type 2 diabetes mellitus in the Middle East and North Africa region. Diabetes Res. Clin. Pract. 149, 18-26 (2019)

20. A. Jabbar, W. Mohamed, R. Ozaki, R. Mirasol, T. Treuer, T. Lew, R. Qi, W.H. Sheu, C. Deerochanawong, S.M. Babineaux, Patterns and trends in insulin initiation and intensification among patients with type 2 diabetes mellitus in the Western Pacific region. Curr. Med. Res. Opin. 34, 1653-1662 (2018)

21. V. Mohan, S. Shah, B. Saboo, Current glycemic status and diabetes related complications among type 2 diabetes patients in India: data from the A1chieve study. J. Assoc. Physicians India 61 (Suppl:), 12-5 (2013) 